\title{
Optical astrometry of Benchmark radio sources
}

\section{New results in the southern hemisphere}

\author{
E. Costa and P. Loyola \\ Departamento de Astronomía, Universidad de Chile. Casilla 36-D Santiago, Chile
}

Received June 11; accepted August 5, 1996

\begin{abstract}
Optical positions relative to the International Reference Stars Catalogue (IRS), and therefore in the system of the FK5, have been obtained for the optical counterparts of 40 compact extragalactic radio sources south of $\delta \sim+6^{\circ}$. Many of these sources are being considered as possible Benchmark objects for the establishment of a quasi-inertial unified radio/optical reference frame. Precision levels as good as $0.1^{\prime \prime}$ in both coordinates were achieved. A comparison with VLBI radio positions available for these sources is presented. We give new evidence for the existence of an offset in the origin of right ascension between the radio and optical reference frames as defined at present.
\end{abstract}

Key words: astrometry — reference systems

\section{Introduction}

In this paper we give new results of a decade-old program to determine precise optical positions for the optical counterparts of compact extragalactic radio sources (CERS) being considered as possible Benchmark objects to link the new high-precision VLBI radio reference frame (see e.g. Johnston et al. 1995, hereafter Johnston), with the optical reference frame. Of the roughly 600 objects that have been or are present being considered as possible link sources (Fey 1994), we have obtained homogeneous optical positions at a precision level as good as $0.1^{\prime \prime}$ in both coordinates for about 250 of them by means of routine observations carried out with a flat-field 70/100/210 $\mathrm{cm}$ Maksutov astrograph. Comparison of our optical data with available VLBI radio positions has allowed us to uncover misidentifications and unsuitable link objects -thus contributing to the basic goal of selecting the most adequate Benchmark sources- and to evaluate the existence of offsets between the radio and the optical frames as defined at present (see e.g. Costa \& Loyola 1996, and references therein).
Here we present new optical observations of 40 southern CERS selected mainly from the list of Jauncey et al. (1989), hereafter Jauncey. The optical positions were measured with respect to the stars of the IRS catalogue (Corbin 1991), and therefore are given in the system of the FK5.

\section{Observations and reductions}

References to the optical identifications, finding charts and other relevant data for the sources observed can be found in Jauncey et al. (1993). Notes on individual objects are given in Sect. 4.

The observations were carried out with the Maksutov astrograph (field: $5^{\circ} \times 5^{\circ}$, scale: $100^{\prime \prime} / \mathrm{mm}$ ) at the Estación Astronómica de Cerro el Roble, operated by the University of Chile. Details on the observational and reduction procedure can be found in Costa \& Loyola (1992, 1994 and 1996; hereafter Papers I, II and III, respectively).

\section{Results and errors}

The results, in the FK5(J2000) system, are presented in Table 1. The first column gives the IAU designation of the sources, the second and fourth columns the resulting optical positions and the third and fifth columns their corresponding total internal errors. Finally, Col. (6) gives the epochs of the observations.

The errors of the measurements were determined as described in Sect. 4.2 of Paper I. The total internal errors of the positions presented in Table 1 were determined using the same relations given in Sect. 4.1 of Paper I. The total error contributed by the IRS catalogue was estimated on a zonal basis, as explained in Paper II.

\section{Notes on individual objects}

1236-684: The optical counterpart proposed by Jauncey -for which we give optical coordinates in Table 1is incorrect. This conclusion is sustained by the high (radio-optical) residuals obtained when comparing our 


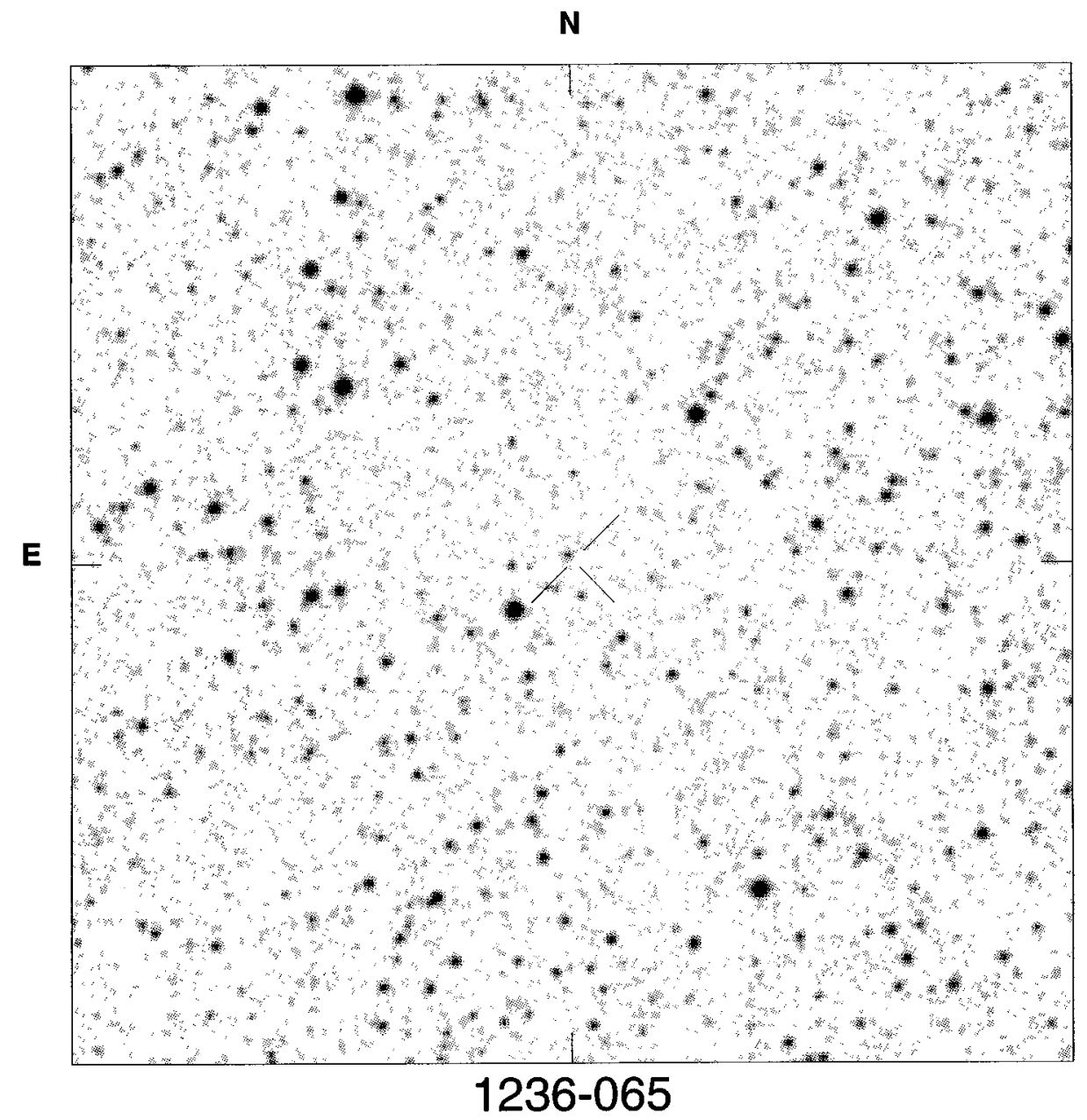

Fig. 1. Finding chart for $1236-684$. Chart is 9 arcmin on a side. See Sect. 4 for details

optical position for this object with the VLBI radio position given by Johnston (see Table 2). As illustrated by Fig. 1, which shows more or less precisely the position of the radio source, the object proposed by Jauncey lies well offset to the NE of the radio source. At the limit of our plates $(B \approx 20.5)$, no faint alternative counterpart is seen. The finding chart and the radio source position identification were obtained using standard IRAF procedures from a digitized image extracted from the Digitized Sky Survey (DSS), produced by the Space Telescope Science Institute. The scan used is based on a 4 min. exposure visual plate taken with the UK Schmidt telescope. The unfortunate fact that this scan is based on a shallow "supplemental" plate of the UK Schmidt southern sky survey plate collection, prevented us from examining the possible existence of a very faint alternative optical counterpart, at a magnitude level of $B \approx 22.5$ (which is the approximate limiting magnitude of the "standard" deep blue UK Schmidt survey plates).

2052-474: The finding chart for this object published by Jauncey is incorrect. A correct identification for the optical counterpart of this source can be found in Murdoch et al. (1984).

2314-409: Identification of the optical counterpart of this source is claimed by Jauncey, but no finding chart has been published. In Fig. 2 we present a tentative optical counterpart for this source, whose coordinates are given in Table 1 . The finding chart and the optical identification were obtained, as explained for Fig. 1, from a DSS scan based on a 60 min. exposure UK Schmidt blue plate.

\section{Comparison with other data and discussion}

Table 2 gives the differences in the FK5(J2000) system with the VLBI radio positions published by Johnston, in the sense radio minus this work (CL). With the exception of the source $1236-684$ (see previous section), the comparison shows a good overall agreement, consistent with the declared precision of the results being compared. Two other sources (0454-463 and 2314+038) for which we give an optical position in Table 1, have been declared unsuccessfully observed by Johnston. As might be expected, a 
Table 1. Optical positions in the FK5 system of 40 compact extragalactic radio sources

\begin{tabular}{|c|c|c|c|c|c|}
\hline (1) & $(2)$ & (3) & (4) & $(5)$ & $(6)$ \\
\hline \multirow{2}{*}{$\begin{array}{c}\text { IAU } \\
\text { Designation }\end{array}$} & $\alpha(\mathrm{J} 2000.0)$ & \multirow{2}{*}{$\begin{array}{c}\epsilon_{\alpha} \cos \delta \\
\pm^{\prime \prime}\end{array}$} & $\delta(\mathrm{J} 2000.0)$ & $\epsilon_{\delta}$ & \multirow{2}{*}{$\begin{array}{l}\text { Plate } \\
\text { Epoch }\end{array}$} \\
\hline & $\mathrm{h} \quad \mathrm{m} \quad \mathrm{s}$ & & $\circ \quad 1$ & $\pm^{\prime \prime}$ & \\
\hline $0019+058$ & 002232.402 & 0.11 & +060803.93 & 0.10 & 89.11 .23 \\
\hline $0034-014$ & 003704.151 & 0.16 & -010908.11 & 0.11 & 93.11 .08 \\
\hline $0035-024$ & 003820.516 & 0.09 & $\begin{array}{lll}-02 & 07 & 40.72\end{array}$ & 0.10 & 93.10 .07 \\
\hline $0131-522$ & 013305.800 & 0.13 & -520003.93 & 0.10 & 92.11 .17 \\
\hline $0219-637$ & 022054.202 & 0.17 & -633019.07 & 0.10 & 93.11 .09 \\
\hline $0332-403$ & 033413.638 & 0.12 & -400825.44 & 0.11 & 94.01 .04 \\
\hline $0355-483$ & 035721.936 & 0.28 & $-48 \quad 12 \quad 15.13$ & 0.09 & 93.01 .17 \\
\hline $0454+039$ & 045847.167 & 0.11 & +034752.43 & 0.09 & 93.01 .15 \\
\hline $0454-463$ & 045550.799 & 0.13 & -461559.00 & 0.13 & 93.01 .16 \\
\hline $0521-262$ & 052318.470 & 0.15 & -261409.52 & 0.09 & 93.01 .18 \\
\hline $0537-158$ & 053932.018 & 0.08 & -155030.23 & 0.13 & 93.01 .16 \\
\hline $0622-441$ & 062331.791 & 0.10 & $-44 \quad 1302.62$ & 0.13 & 93.01 .15 \\
\hline $1202-262$ & 120533.254 & 0.13 & -263404.15 & 0.12 & 93.06 .18 \\
\hline $1206-399$ & 120935.247 & 0.13 & -401613.31 & 0.12 & 92.05 .02 \\
\hline $1236-684$ & 123947.047 & 0.20 & -684529.28 & 0.12 & 93.06 .15 \\
\hline $1351+021$ & 135351.590 & 0.09 & +015153.27 & 0.09 & 93.06 .18 \\
\hline $1509+022$ & 151215.745 & 0.08 & +020317.01 & 0.11 & 93.06 .15 \\
\hline $1549-790$ & 155658.983 & 0.30 & $-79 \quad 1404.14$ & 0.11 & 93.06 .16 \\
\hline $1602-001$ & 160456.142 & 0.12 & -001906.85 & 0.12 & 93.06 .14 \\
\hline $1603+001$ & 160612.702 & 0.13 & +00 0026.99 & 0.11 & 93.06 .15 \\
\hline $1718-649$ & 172340.937 & 0.18 & -650036.47 & 0.17 & 93.06 .14 \\
\hline $1733-565$ & 173735.773 & 0.15 & -563403.27 & 0.13 & 93.06 .17 \\
\hline $1823-455$ & $\begin{array}{lll}18 & 27 & 10.246\end{array}$ & 0.13 & -453309.50 & 0.16 & 93.06 .17 \\
\hline $1831-711$ & 183728.706 & 0.29 & -710843.36 & 0.10 & 93.07 .12 \\
\hline $1852-534$ & 185700.441 & 0.14 & -532500.40 & 0.12 & 93.07 .12 \\
\hline $1929-457$ & 193244.885 & 0.12 & -453637.94 & 0.09 & 93.07 .20 \\
\hline $2004-447$ & 200757.084 & 0.13 & -443446.85 & 0.16 & 93.07 .12 \\
\hline $2022-702$ & 202724.608 & 0.20 & $-7007 \quad 17.15$ & 0.09 & 92.09 .24 \\
\hline $2052-474$ & 205616.376 & 0.15 & $-47 \quad 1447.52$ & 0.13 & 92.09 .25 \\
\hline $2058-425$ & 210159.127 & 0.11 & -421916.66 & 0.10 & 93.09 .11 \\
\hline $2105-489$ & 210850.768 & 0.12 & -484621.57 & 0.09 & 93.09 .12 \\
\hline $2205-636$ & 220847.322 & 0.13 & -632547.16 & 0.10 & 92.09 .25 \\
\hline $2217+018$ & 222030.771 & 0.09 & +020453.34 & 0.11 & 93.09 .11 \\
\hline $2314-409$ & 231646.937 & 0.15 & -404121.27 & 0.16 & 92.09 .26 \\
\hline $2324+038$ & 231635.232 & 0.10 & +040518.06 & 0.09 & 93.09 .12 \\
\hline $2324-023$ & 232653.804 & 0.10 & $-02 \quad 02 \quad 14.14$ & 0.09 & 93.09 .11 \\
\hline $2327-459$ & 233037.665 & 0.09 & -453958.01 & 0.08 & 93.11 .09 \\
\hline $2332-017$ & 233520.386 & 0.11 & -013110.04 & 0.11 & 93.11 .10 \\
\hline $2351-006$ & 235409.180 & 0.08 & -001948.11 & 0.11 & 93.10 .07 \\
\hline $2352-455$ & 235528.785 & 0.11 & -451325.49 & 0.11 & 93.10 .06 \\
\hline
\end{tabular}

comparison of their a-priori radio positions given in the NRL/USNO radio/optical survey working list (Fey 1994) with our optical data gives very high residuals, and therefore were not included in Table 2 . For both objects optical positions have been obtained by Jauncey, in the FK4 system. In the case of $0454-463$ the optical position given by Jauncey (transformed to the FK5, J2000 system) is in good agreement with ours. As pointed out by Johnston, the radio counterpart was apparently correlated at the wrong position. As for $2314+038$, our optical position is not consistent with Jauncey's.

Combining the results presented in Table 2 with analogous results published in Papers II and III of our series, a comparison subset of 45 objects with the VLBI positions given by Johnston is obtained. After the omission of the 


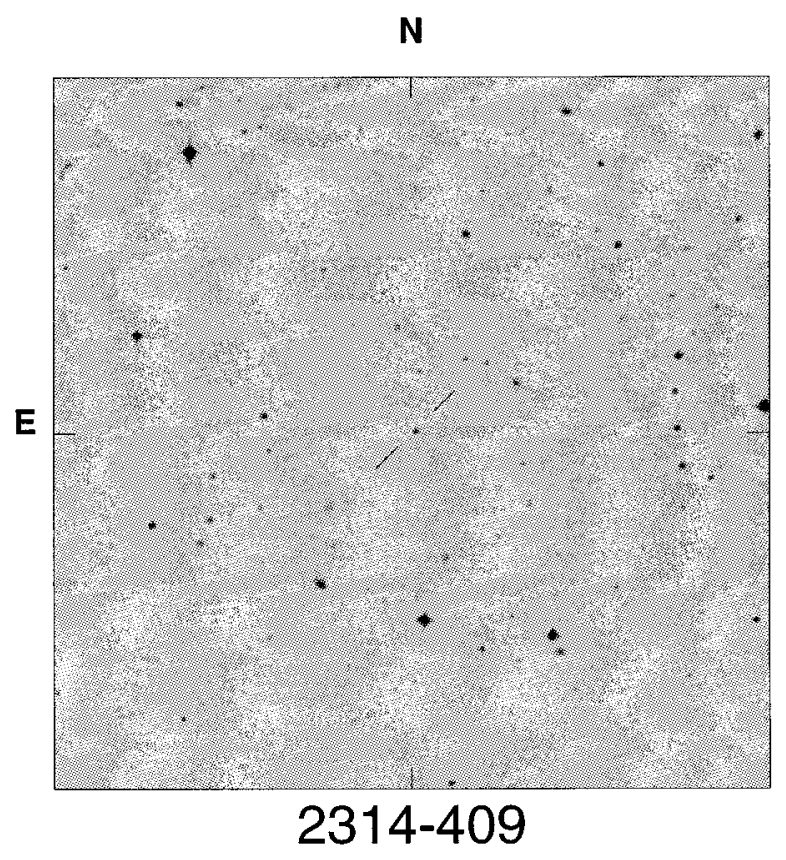

Fig. 2. Finding chart for $2314-409$. Chart is 9 arcmin on a side. See Sect. 4 for details

Table 2. Comparison in the FK5 system with the VLBI radio positions given by Johnston et al. (1996)

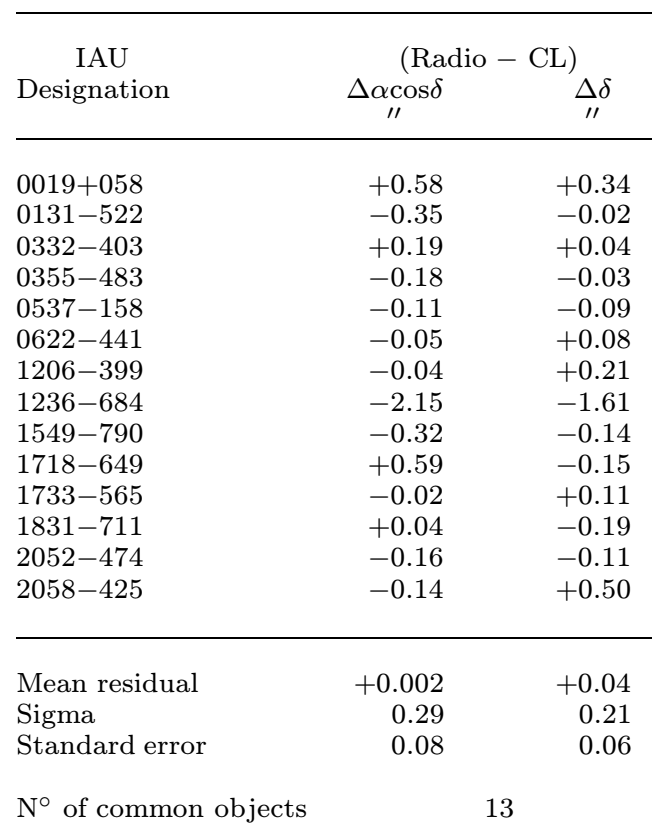



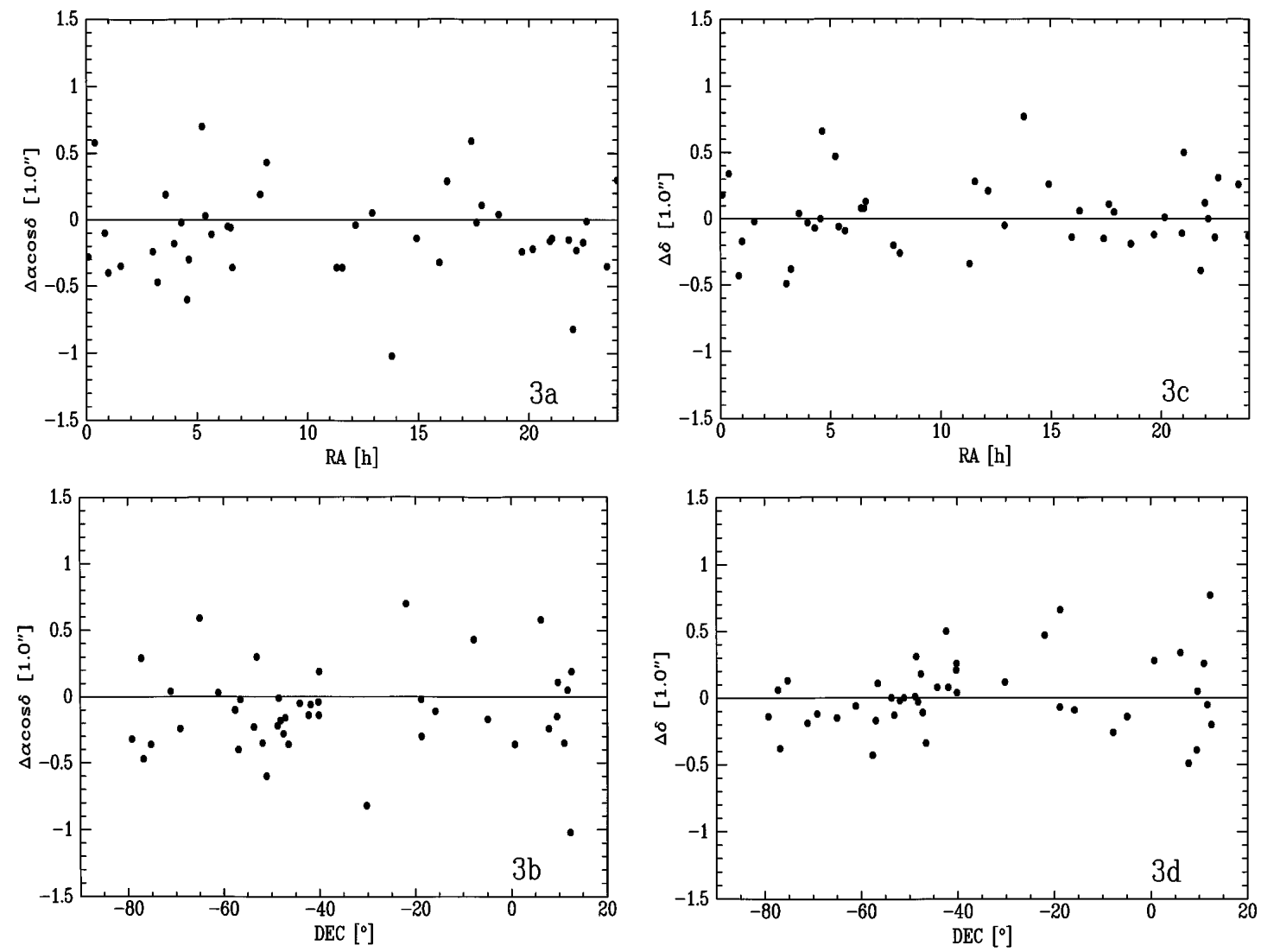

Fig. 3. a-d) Residuals in the sense (radio - CL), plotted as a function of the coordinates, for the 43 sources in common between the VLBI survey of Johnston et al. (1995) and the optical survey of Costa \& Loyola (Papers II, III and IV). See Sect. 5 for details

de Investigación Científica y Tecnológica (proyecto 1930784, Fondecyt).

\section{References}

Argyle R.W., Einicke O.H., Pilkington J.D.H., et al., 1996, A\&A (in press)

Corbin T.E., 1991, Internat. Ref. Stars Cat., Washington, U.S. Naval Observatory

Costa E., Loyola P., 1992, A\&AS 96, 183 (Paper I)

Costa E., Loyola P., 1994, A\&AS 104, 503 (Paper II)
Costa E., Loyola P., 1996, A\&AS 115, 75 (Paper III)

Fey A.L., 1994, The NRL/USNO proposed reference frame (private communication)

Jauncey D.L., Savage A., Morabito D.D., et al., 1989, AJ 98, 54

Johnston K.J., Fey A.L., Zacharias N., et al., 1995, AJ 110, 880

Murdoch H.S., Hunstead R.W., White G.L., 1984, Proc. ASA 5, 341

Véron-Cetty M.P., Véron P., 1993, E.S.O. Scientific Report $\mathrm{N}^{\circ} 13$ 